Revista Brasileira de Odontologia Legal - RBOL

\title{
Identificação odontológica
}

\section{IMPORTÂNCIA DO REGISTRO DAS AUSÊNCIAS DENTAIS PARA A IDENTIFICAÇÃO HUMANA: RELATO DE CASO.}

\section{Importance of the registration of dental absences for human identification: case report.}

Adriana de Moraes CORREIA ${ }^{1}$, Débora da Silva BARBOSA ${ }^{2}$, Jeisiana Alves da Silva ALCANTARA ${ }^{2}$, Erick Helton Lima FONTENELE ${ }^{3}$, Tácio Pinheiro BEZERRA ${ }^{4}$.

1. Professora do Curso de Mestrado e de Graduação em Odontologia, Área Odontologia Legal, Centro Universitário Christus, Ceará Brasil.

2. Cirurgiã-Dentista, Graduada pelo Centro Universitário Christus, Ceará Brasil.

3. Aluno do Programa de Mestrado Acadêmico em Odontologia, Área Clínica Odontológica, Centro Universitário Christus ,Ceará Brasil.

4. Professor do Curso de Graduação em Odontologia, Área Odontologia Legal e Cirurgia e Traumatologia Buco-MaxiloFacial, Odontologia, Centro Universitário Christus, Ceará Brasil.

Informação sobre o manuscrito

Recebido em: 07 Nov 2019

Aceito em: 19 Dez 2019
Autor para contato:

Prof. Tácio Pinheiro Bezerra.

Rua Israel Bezerra, 1033, Apartamento 501, Bairro São João do Tauape. Fortaleza, Ceará, Brasil. 60.135-460

E-mail: taciopb@gmail.com.

\begin{abstract}
RESUMO
Identificar é determinar ou comprovar a identidade de algo, alguém ou de si mesmo, para isso é necessário um conjunto de procedimentos e diligências. O processo de identificação pode ser realizado por diferentes métodos, em casos de cadáveres carbonizados, muitas vezes, o método de escolha para a identificação é o comparativo entre as particularidades odontológicas ante e post mortem. O objetivo deste trabalho é relatar um caso de identificação humana de corpo carbonizado através do método de comparação odontológica. No necrotério do Instituto de Perícias Oficiais local, deram entrada três corpos carbonizados envolvidos em sinistro aeronáutico. O perito odontolegista foi solicitado para participar do processo de identificação de umas das vítimas. O procedimento pericial consistiu na elaboração de um odontograma específico para o corpo não identificado (exame post-mortem) e confronto com os documentos assistenciais (prontuário odontológico) apresentados como sendo da vítima envolvida no acidente (exame ante-mortem). Após a uniformização dos dados Ante Mortem e Post Mortem, os odontogramas foram confrontados gerando o resultado positivo para identificação, destacando-se o registro de várias ausências dentais. Frente ao relato do caso, destaca-se a importância da atuação do cirurgião-dentista no contexto pericial, contribuindo significativamente com a efetividade dos exames realizados, com a celeridade dos resultados, oferecendo uma opção de baixo custo e alta eficiência para a identificação de corpos carbonizados, dispensando outros métodos de identificação.
\end{abstract}

PALAVRAS-CHAVE

Odontologia legal; Identificação humana; Registros odontológicos.

\section{INTRODUÇÃO}

Identificar, do ponto de vista pericial, é determinar ou comprovar a identidade de algo, alguém ou de si mesmo, para isso é necessário um conjunto de procedimentos $\mathrm{e}$ diligências. 
Em alguns Órgãos de Perícias Oficiais brasileiros há a presença do setor de Antropologia Forense, o qual geralmente é formado por uma equipe multidisciplinar de peritos especializados e treinados sendo eles normalmente: médicos-legistas, peritos criminais e peritos odontolegistas ${ }^{1-2}$.

O processo de identificação inclui diferentes técnicas que buscam encontrar os caracteres próprios e exclusivos de uma pessoa. A identificação humana pela análise das impressões digitais é um método bastante prático. Porém, em casos de cadáveres carbonizados, fragmentados e, muitas vezes, em estado avançado de decomposição, o odontolegista tem um papel fundamental na identificação destes ${ }^{1}$.

O método odontológico constitui um dos melhores e mais eficientes métodos para identificação humana, tendo em vista que os dentes são as estruturas mais estáveis e resistentes do corpo. Os dentes ainda fornecem informações particulares de um indivíduo, graças a seus posicionamentos, interferências terapêuticas e demais singularidades obtidas durante a vida, tornando o método confiável, fácil e rápido ${ }^{3}$.

Em procedimentos de identificação humana, a Odontologia pode contribuir com diferentes métodos como identificação pelos arcos dentais, dna, rugoscopia palatina, fotografias do sorriso, determinação do sexo pelas características cranianas, estimativa da idade pelos dentes e também contribuir com a necropsia virtual ${ }^{4}$.

Dentre tais métodos destaca-se o de comparação que consiste em confrontar achados odontológicos registrados em vida (antes da morte - AM) com os constatados em cadáveres (pós-morte - PM) e tal método mostra-se eficiente $\mathrm{e}$ de baixo custo $^{5-6}$. No entanto, a possibilidade de se obter resultados satisfatórios depende da qualidade e quantidade de informações contidas na documentação odontológica apresentada e também da relevância das informações coletadas no exame do corpo que se quer identificar ${ }^{7}$.

A comparação em busca de um resultado para identificação odontolegal é baseada, em especial, nos dentes e nas restaurações presentes. No entanto, podese considerar também as ausências dentais, alterações de posicionamento de dentes e as condições de erupção. No aspecto das bases ósseas, apresentação de sinais anatômicos podem também servir de referenciais para identificação. Desta forma, os achados são fundamentadas em alterações da morfologia/anatomia das estruturas analisadas, bem como, nas alterações patológicas experimentadas e seus possíveis tratamentos aplicados ${ }^{8}$. Mas também, existe a possibilidade da utilização de informações coletados indiretamente de exames tomados da pessoa que será fonte de informações, tais como modelos de gesso para confecção de próteses dentárias ${ }^{9}$.

$O$ presente relato de caso se faz importante pelo fato de que a atuação da Odontologia Legal dentro do contexto pericial demonstra-se sólida e eficaz, especialmente quando os registros odontológicos são adequadamente confeccionados. Neste contexto, o presente trabalho tem como objetivo demonstrar a importância dos registros odontológicos para a identificação humana, especialmente 
de ausências dentais, por meio de um relato de caso pericial.

Este relato de caso foi submetido e aprovado por Comitê de Ética em PesquisaCEP sob Parecer № 2.959.657 (CAAE 98514918.0.0000.5049).

\section{RELATO DO CASO}

Três corpos carbonizados envolvidos em sinistro aeronáutico deram entrada no necrotério de um Instituto de Perícias Oficiais, sendo a equipe de perícia odontolegal acionada para participar do processo de identificação de uma das vítimas.

Para tanto, foi solicitado que os supostos familiares da vítima apresentassem documentos ou registros odontológicos produzidos antes do acidente, tais como: prontuários odontológicos, registros radiográficos, fichas de inspeção em saúde ou qualquer outro documento que fosse fonte de dados para identificação odontolegal $^{7-10}$, os quais serviriam como registros Ante Mortem (AM).

O resultado desta busca foi efetivo, sendo apresentado um formulário onde constava "Exame e Identificação Odontológica”, que continha um odontograma (Figura 1) com marcações de procedimentos, dentes ausentes e outras informações que foram compiladas na Tabela 1.

Tendo em vista a presença de registro odontológico produzido em vida e com potencial para identificar a vítima, foi realizado 0 exame post-mortem no necrotério do Instituto de Perícias Oficiais local, seguindo-se o seguinte fluxo:

- Fotografia do corpo.
- Descrição pormenorizada das vestes, adornos e outros pertences.

- Minucioso exame cadavérico externo, com a limpeza das sujidades na superfície cutânea para a pesquisa de eventuais cicatrizes, tatuagens ou marcas na pele.

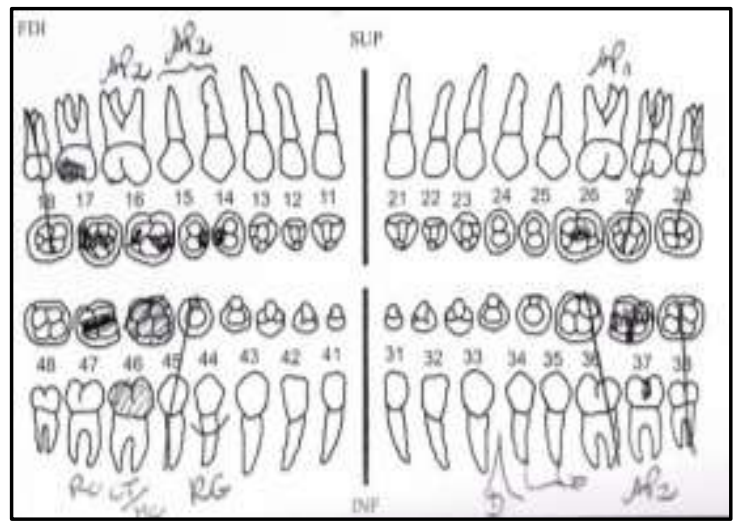

Figura 1 - Odontograma contendo as informações AM.

Legenda: $\mathrm{AP}_{1}$ : Amálgama de prata (1 face); $\mathrm{AP}_{2}$ : Amálgama de prata (2 faces); D: diastema; RC (resina composta); CT/MC (coroa total/metalocerâmica); RG: Recessão Gengival.

Para a realização do exame odontolegal pós-morte, considerando 0 estágio de carbonização do corpo, foi realizado o acesso necroscópico conforme descrito por Stimson e Mertz (1997) ${ }^{8}$, permitindo expor os arcos dentais em norma frontal e lateral - Figura 2. Após esse processo, foram evidenciadas particularidades odontológicas anatômicas e terapêuticas, especialmente ausências dentais nos quatro hemiarcos que foram também compiladas na Tabela 1.

Após a uniformização dos dados Ante Mortem e Post Mortem, foram confeccionados dois odontogramas (AM e PM - Figuras 3 e 4), conforme o Guia de Identificação de Vítimas de Desastres da 
Interpol, cujos dados foram confrontados em busca de similaridades e discrepâncias (explicáveis e inexplicáveis) que pudessem permitir (ou não) a identificação da vítima ${ }^{10-}$ 12.

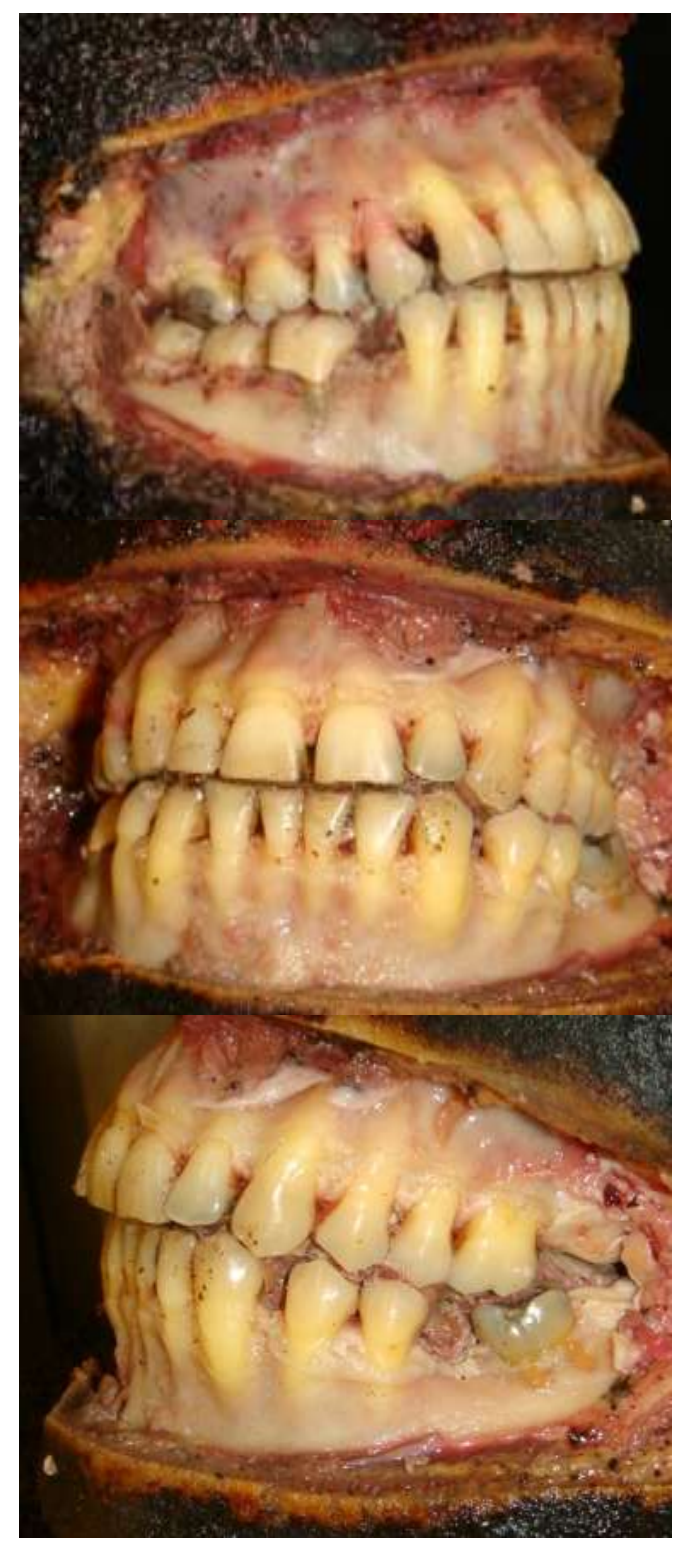

Figura 2 - Vistas frontal e laterais pós-morte, após remoção dos tecidos moles bucais.

Observou-se, por meio do confronto odontolegal, que um total de 15 pontos relevantes de confronto foram identificados, estando relacionados com as ausências dos dentes 18, 27, 28, 36, 38 e 45, diastema entre os dentes 33 e 34 , além das coincidências das restaurações de amálgama presentes nos dentes $14,15,16$, 17,26 e 37 , resina composta no dente 47 como também a prótese fixa do dente 46 . Estes pontos de similaridades permitiram chegar à conclusão de o corpo carbonizado pertencia à pessoa desaparecida, configurando uma identificação positiva.

\section{DISCUSSÃO}

Um adequado método de
identificação humana é aquele que preenche requisitos técnicos e biológicos. Os técnicos dizem respeito à capacidade de o método ser classificável e quanto à sua praticabilidade, avaliando custo, facilidade na obtenção de registro, materiais disponíveis, dentre outros. Já os biológicos se referem às características das informações utilizadas, tais como: unicidade das características; imutabilidade dos registros no decorrer do tempo; e perenidade que é a capacidade de resistir às agressões do tempo ${ }^{13}$.

De acordo com Guia de identificação de vítimas de desastres (DVI) da Interpol, as metodologias disponíveis para a identificação humana podem ser agrupadas em métodos primários e secundários. Os primários são aqueles suficientes para levar à identificação de uma pessoa, composto pela análise de impressões digitais, a análise odontológica e estudo do perfil de DNA. Os métodos secundários de identificação incluem a descrição pessoal, os dados médicos, assim como as evidências e roupas encontradas no corpo e eles servem como direcionamento da identificação, sem, no entanto, serem suficientes para resultar na identidade ${ }^{7}$. 
Tabela 1 - Confronto entre os achados obtidos nos exames Ante e Post Mortem.

\begin{tabular}{|c|c|c|c|}
\hline Dente $^{*}$ & Registro AM & Registro PM & Confronto \\
\hline 11 & Sem registro & Hígido & Prejudicado \\
\hline 12 & Sem registro & Hígido & Prejudicado \\
\hline 13 & Sem registro & Hígido & Prejudicado \\
\hline 14 & RA & RA & Similaridade \\
\hline 15 & RA & RA & Similaridade \\
\hline 16 & RA & RA & Similaridade \\
\hline 17 & RA & RA & Similaridade \\
\hline 18 & Ausência dental & Ausência dental & Similaridade \\
\hline 21 & Sem registro & Hígido & Prejudicado \\
\hline 22 & Sem registro & Hígido & Prejudicado \\
\hline 23 & Sem registro & Hígido & Prejudicado \\
\hline 24 & Sem registro & Hígido & Prejudicado \\
\hline 25 & Sem registro & Hígido & Prejudicado \\
\hline 26 & $\mathrm{RA}$ & RA & Similaridade \\
\hline 27 & Ausência dental & Ausência dental & Similaridade \\
\hline 28 & Ausência dental & Ausência dental & Similaridade \\
\hline 31 & Sem registro & Hígido & Prejudicado \\
\hline 32 & Sem registro & Hígido & Prejudicado \\
\hline 33 & Sem registro & Hígido & Prejudicado \\
\hline 34 & Sem registro & Hígido & Prejudicado \\
\hline 35 & Sem registro & Hígido & Prejudicado \\
\hline 36 & Ausência dental & Ausência dental & Similaridade \\
\hline 37 & RA & $\mathrm{RA}$ & Similaridade \\
\hline 38 & Ausência dental & Ausência dental & Similaridade \\
\hline 41 & Sem registro & Hígido & Prejudicado \\
\hline 42 & Sem registro & Hígido & Prejudicado \\
\hline 43 & Sem registro & Hígido & Prejudicado \\
\hline 44 & Sem registro & Hígido & Prejudicado \\
\hline 45 & Ausência dental & Ausência dental & Similaridade \\
\hline 46 & Coroa metalo-cerâmica & Coroa metalo-cerâmica & Similaridade \\
\hline 47 & $\mathrm{RC}$ & $\mathrm{RC}$ & Similaridade \\
\hline 48 & Sem registro & Hígido & Prejudicado \\
\hline
\end{tabular}

Legenda: RA: restauração em amálgama; $\mathrm{RC}$ : restauração em resina composta.

*Notação dental preconizada pela FDI. 


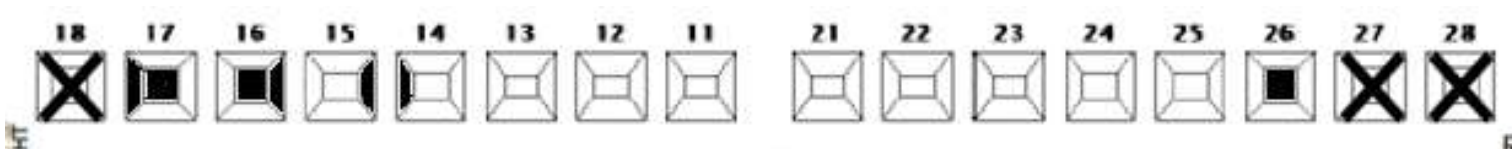
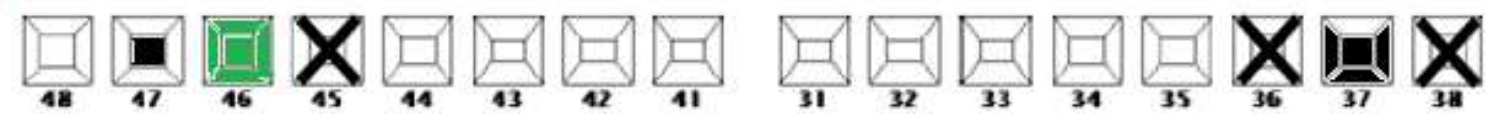

Figura 3 - Registro do exame Ante Mortem. Preenchido conforme o guia DVI Interpol.

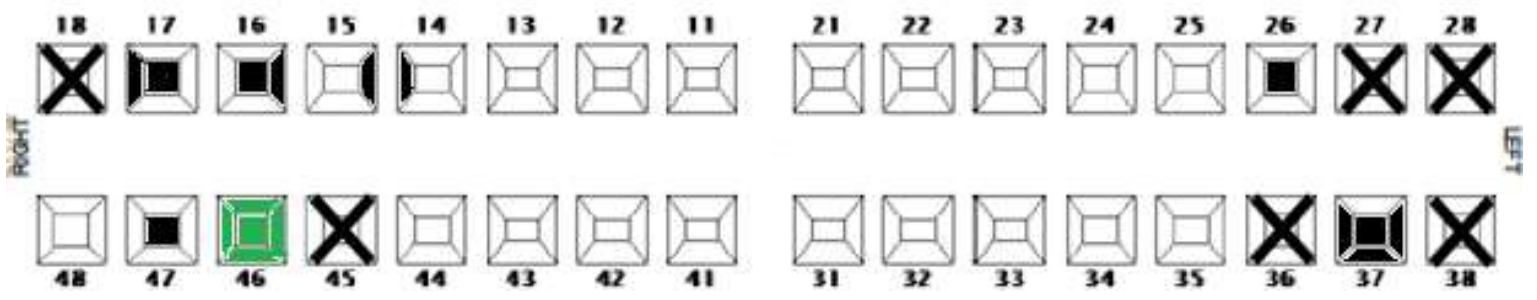

Figura 4 - Registro do exame Post Mortem. Preenchido conforme o guia DVI Interpol.

Além de tais critérios, deve-se levar em consideração, na escolha do método de identificação humana, o estado do cadáver (putrefeito; carbonizado; esqueletizado; mumificado) ${ }^{14,15}$. No caso apresentado, por se tratar de um acidente que culminou na carbonização do corpo inviabilizando a realização do exame datiloscópico, a identificação positiva do individuo só foi possível através do método odontológico comparativo, que se demonstra eficiente tendo em vista que os dentes $e$ as restaurações odontológicas são extremamente resistentes à destruição pelo fogo $0^{16}$.

Para obtenção de toda informação dental no exame post mortem, o acesso necroscópico é um ponto relevante que compete ao odontolegista a escolha da forma mais viável de acesso, que dependerá do grau de rigidez cadavérica ou da aderência dos músculos faciais aos ossos nos casos de carbonização $0^{3,8,17}$.

Seguido do devido acesso, o preenchimento do odontograma é fundamental, e foi feito de acordo com o
Guia de Identificação de Vítimas de Desastres da Interpol que tem um padrão mundial referencial, segundo 0 seu manual $^{7,10,18}$. Esse guia é muito utilizado em desastres em massa, que acometem um número maior de vítimas, tendo o intuito de oferecer uma resposta rápida e eficiente. No odontograma AM coletado da vítima observa-se que tal padrão não foi seguido, porém evidencia-se que o profissional teve 0 cuidado em reproduzir o contorno, a extensão e a delimitação das restaurações efetuadas, principalmente do dente 17, 37 e a coroa fixa do dente 46 , o que facilitou durante 0 confronto individual para estabelecimento da identificação positiva. Considerando a interpretação de odontogramas $\mathrm{AM}$, os símbolos, codificações e terminologias dentais podem variar muito, criando assim a tarefa difícil e demorada de decodificação e interpretação de informações para posteriores comparações $^{18}$, nesses casos evidencia-se a importância do protocolo facilitando a comparação e estando menos sujeita ao erro humano. 
Entretanto, a obtenção de resultados satisfatórios depende diretamente da quantidade e da qualidade das informações contidas na documentação odontológica, especialmente as que devem ser registradas pelo profissional durante 0 exame clínico, no plano de tratamento e após a execução dos procedimentos (evolução do tratamento) ${ }^{11}$. Erros de preenchimento de fichas clínicas, falta de anotações ou anotações errôneas podem conduzir a impossibilidades de identificação ${ }^{10,19}$. Ressalta-se que se trata de um dever ético o profissional da Odontologia manter adequadamente arquivada toda a documentação odontológica produzida em função do tratamento de seus pacientes, conforme preconiza o Artigo 5으 (VIII) do Código de Ética Odontológica ${ }^{6}$.

\section{CONSIDERAÇÕES FINAIS}

A identificação humana por meio da análise odontológica é de grande utilidade nas Ciências Forenses e, portanto, prontuários e fichas decorrentes de tratamentos clínicos devem sempre estar organizados e atualizados. No estudo de caso apresentado, comprova-se a eficácia do método odontológico e a importância da documentação devidamente preenchida do paciente realizada pelo cirurgião-dentista com uma riqueza de detalhes (destacandose as ausências dentais e restaurações), proporcionando maior robustez na identificação positiva. Além disso, fica destacada a importância do odontolegista para realização do exame odontológico com vistas à identificação humana em serviços de pericias oficiais de natureza criminal.

\begin{abstract}
To identify is to determine or prove the identity of something, of someone or of oneself; for this, a set of procedures and diligences are necessary. The identification process can be performed by different methods; in cases of charred corpses, the method of choice for identification is often the comparative between dental and post-mortem dental characteristics. The objective of this work is to report a case of human identification of charred body by means of the method of dental comparison. In the morgue of the local Forensic Institute, three carbonized bodies involved in an aeronautical accident were admitted. A forensic dentist was asked to participate in the process of identifying one of the victims. The expert procedure consisted in the elaboration of a specific dental chart for the unidentified body (post-mortem examination) and confrontation with the assistance documents (dental records) presented as being of the victim involved in the accident (ante-mortem examination). After the standardization of Ante- Mortem and Post-Mortem data, the dental charts were confronted, generating the positive result for identification, highlighting the record of several dental absences. In view of the case report, the importance of the dental surgeon's performance in the expert context is of extreme value, contributing significantly to the effectiveness of the exams performed, with the speed of results, offering a low cost and high efficiency option for the identification of bodies rather than other methods of identification.
\end{abstract}

\title{
KEYWORDS
}

Forensic dentistry; Human identification; Dental records.

\section{REFERÊNCIAS}

1. Terada ASSD, Leite NLP, Silveira TCP, Secchieri JM, Guimarães MA, Silva RHA. Identificação Humana em Odontologia Legal por meio de registro fotográfico de sorriso: relato de caso. Rev Odontol UNESP. 2011; 40(4):199-202.

2. Scolarick RA, Barbieri AA, Moraes ZM, Júnior LF, Júnior ED, Naressi SCM. Identificação humana por meio do estudo de imagens radiográficas odontológicas: relato de caso. Rev Odontol UNESP. 2013; 42(1):67-71. Disponível em: http://dx.doi.org/10.1590/S180725772013000100012.

3. Ribas-e-Silva V, Terada ASSD, Silva RHA. A importância do conhecimento especializado do cirurgião-dentista nas equipes de perícia oficial do Brasil. Rev 
Bras Odontol Leg RBOL. 2015; 2(1):68-90. http://dx.doi.org/10.21117/rbol.v2i1.22.

4. Coutinho CGV, Ferreira CA, Queiroz LR, Gomes LO, Silva LA. O papel do odontolegista nas perícias criminais. RFO. 2018 setembro-dezembro; 18(2):217-223. Disponível em: https://doi.org/10.5335/rfo.v18i2.3399.

5. Zilio F, Basualdo A, Cruz RA. Meios de identificação odontolegal. - VII Mostra de Iniciação Científica e Extensão Comunitária e VI Mostra de Pesquisa de Pós-Graduação. IMED. Passo Fundo: Anais: 2013. p. 1-10.

6. Silva RF, Chaves P, Paranhos LR, Lenza $M A$, Júnior ED. Utilização de documentação ortodôntica na identificação humana. Dental Press J Orthod. 2011;16(2):52-57. Disponível em: http://dx.doi.org/10.1590/S217694512011000200007

7. Interpol. Disater Victim Identification Guide, 2018. Disponível em: https://www.interpol.int/How-wework/Forensics/Disaster-VictimIdentification-DVI.

8. Stimson PG, Mertz CA. Forensic Odontologia. CRC Press: Boca Raton. 1997.

9. Silva RF, Felter $M$, Tolentino PHMP, Rodrigues LG, Andrade MGBA, Palomeque AMP, Sassi C. Importância Pericial de los Modelos de Yeso Odontológicos para la Identificación de Cuerpo Putrefacto - Relato de Caso. Int. J. Odontostomatol. 2017; 11(3), 273-278. http://dx.doi.org/10.4067/S0718$381 \times 2017000300273$.

10. Biancalana RC, Vieira MGDM, Figueiredo BMJ, Vicente SAF, Dezem TU, Silva RHA. Desastres em massa: a utilização do protocolo de dvi da interpol pela odontologia legal. Rev Bras Odontol Leg RBOL. 2015; 2(2):48-62. http://dx.doi.org/10.21117/rbol.v2i2.38.

11. Silva RF, Prado MM, Barbieri AA, Daruge Júnior $E$. Utilização de registros odontológicos para identificação humana. Revista Sul-Brasileira de Odontologia RSBO. 2009. [acesso 20 de outubro de 2018]; 6(1). Disponível em: http://www.redalyc.org/pdf/1530/15301363 6013.pdf.

12. Araujo LG, Biancalana RC, Terada ASSD, Paranhos LR, Machado CEP, Silva RHA. A identificação humana de vítimas de desastres em massa: a importância e o papel da Odontologia Legal. RFO [Internet]. 15 jan.2014 [acesso 19 novembro 2018]; 18(2). Disponivel em: http://seer.upf.br/index.php/rfo/article/view/ $\underline{3376 .}$
13. Borges LC, Rosa TSA, Dietrich L, Prudente MS, Mota MOA, Andrade CMO. Identificação humana post-mortem por meio da odontologia: Revisão de literatura. Revista de odontologia contemporânea. 2018 Maio; 2(1) 24-27.

14. Andrade L. Aplicação dos métodos de identificação humana post mortem no IML Estácio de Lima no período de janeiro de 2011 a dezembro de 2015. Perspec Medicina Legal Per Med. 2017; 4. Disponível em: http://perspectivas.med.br/2017/10/aplicac ao-dos-metodos-de-identificacao-humanapost-mortem-no-iml-estacio-de-lima-noperiodo-de-janeiro-de-2011-a-dezembrode-2015-2.

15. Lages VA, Pinto PHV, Barros AVN, Andrade ACB, Carvalho JBL, Trajano RKN. A importância da documentação odontológica de usuários de drogas institucionalizados para a identificação post mortem: relato de caso. Rev Bras Odontol Leg RBOL. 2017;4(3):101-10. http://dx.doi.org/10.21117/rbol.v4i3.115.

16. Albuquerque Neto $A D$, Farias Neto $A M$, Cavalcante JRD, Cavalcante DKF, Sampaio TRC, Costa VS. Efeito das altas temperaturas aos tecidos bucodentais e materiais odontológicos: revisão de literatura. Rev Bras Odontol Leg RBOL. 2015; 2(2):89-104. http://dx.doi.org/10.21117/rbol.v2i2.28.

17. Menon LML, Fernandes MM, Paranhos LR, Silva RHA. Tanatologia forense e odontologia legal: interface e importância na rotina pericial. Odonto 2011 ; 19 (37):1523.

18. Mânica S. Outros desafios além da identificação de vítimas para o dentista forense que atua em desastres em massa - considerações em literatura. Rev Bras Odontol Leg RBOL. 2016; 3(1):60-69. http://dx.doi.org/10.21117/rbol.v3i1.53.

19. Almeida SM, Delwing F, Azevedo JAP, Nogueira RKT, Falcão FP, Carvalho SPM. Effectiveness of dental records in human identification. RGO. 2015; 63(4):502-6. http://dx.doi.org/10.1590/1981863720150003000213017. 